\title{
Making algorithms work for us
}

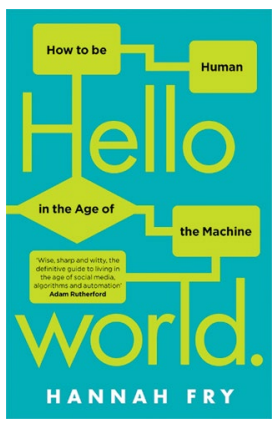

with insufficient data will create serious unintended consequences.

As Fry explains, trusting algorithms to run our lives is made all the more dangerous by the fact that the huge amounts of personal data we hand over to companies and agencies can be used to infer information that many of us would rather keep private. Even data that we think of as innocent enough, such as our grocery habits, can be used to extract valuable information. The book is filled with fascinating descriptions of these cases, including the fact that pregnant women in their second trimester are more likely to buy moisturizer, effectively signalling to a store (via their data analysts) that a baby is due in a few months.

Several chapters of the book are dedicated to explaining the diverse areas where data processing and algorithmic analysis is being applied, including transportation, medicine, justice and crime prevention. Fry analyses how algorithms can be used, for example, to drive cars, influence elections, diagnose cancer and recognize criminals. The examples Fry provides are often riveting. But more importantly, she uses them to highlight what I feel is the central message of the book: using an algorithm is a trade-off and every application brings with it benefits and risks, which have to be weighed. If we use face recognition algorithms to spot criminals, we have to accept the risk of an algorithm sending an innocent person to jail. If we can use social data to target sales campaigns, we have to accept the risk of social data being used to market political candidates and manipulate elections. The list of trade-offs goes on and on, and every one of them is complex.

In engineering there is no such thing as $100 \%$ reliability or $100 \%$ precision. Every system that is designed to perform a specific task will have a probability of failure, however small - and this is no different when engineering algorithms. An autonomous car, for example, may in general be safer than a normal car with a human driver, but it will, in rare cases, cause accidents that would not have happened otherwise. How many machine-made accidents are we willing to tolerate in order to make the roads safer for everyone? These are difficult questions, and the book does a good job of reminding us that technology will not make those choices for us. It is our responsibility to assess and evaluate the benefits and risks of each application of algorithms, and decide what we, as a society, think will make a positive difference in the world.

The final chapter of the book addresses a different, subtler point: can computers be truly creative? Fry does not try to find a final answer to this conundrum, but she provides some interesting data on the subject, for the readers to decide for themselves. By analysing the patterns of the music written by a composer, algorithms can create new pieces that can fool people, and even experts, into believing they were written by a human. Does this mean that computers can produce art? And if so, is it any good? The question is made more difficult by the fact that there are, of course, no objective measures to quantify the quality of works of art. For many, beauty is in the eye of the beholder, and computerproduced art is good enough to be treated like the real thing, at least when the origin of the work is not known. But many will argue that copying someone else's style is not really creating art; others will disagree. This final chapter provides an interesting introduction to the problem.

Fry's clear understanding of the issues surrounding the use of algorithms, and her ability to explain, in simple terms, what are sometimes complex concepts, make Hello World simultaneously engaging for the expert practitioner and accessible to the general reader. For me, only one thing was missing: a discussion of the way things are likely to evolve in the near future, as more data becomes available and algorithms become even more powerful. Perhaps a topic for Fry's next book?

\section{Reviewed by Arlindo Oliveira}

Instituto Superior Técnico, Lisbon, Portugal. e-mail:arlindo.oliveira@cg.tecnico.ulisboa.pt

Published online: 13 September 2018 https://doi.org/10.1038/s41928-018-0138-5 